\title{
Characterization of Negative Exponential Distribution through Expectation*
}

\author{
Milind Bhatt B. \\ Department of Statistics, Sardar Patel University, Vallabh Vidyanagar, India \\ Email: bhattmilind_b@yahoo.com
}

Received May 4, 2013; revised June 4, 2013; accepted June 11, 2013

Copyright (C) 2013 Milind Bhatt B. This is an open access article distributed under the Creative Commons Attribution License, which permits unrestricted use, distribution, and reproduction in any medium, provided the original work is properly cited.

\begin{abstract}
For characterization of negative exponential distribution one needs any arbitrary non-constant function only in place of approaches such as identical distributions, absolute continuity, constant regression of order statistics, continuity and linear regression of order statistics, non-degeneracy etc. available in the literature. Path breaking different approach for characterization of negative exponential distribution through expectation of non-constant function of random variable is obtained. An example is given for illustrative purpose.
\end{abstract}

Keywords: Characterization; Negative Exponential Distribution

\section{Introduction}

Knowing characterizing property may provide unexpectedly accurate information about distributions and one can recognize a class of distributions before any statistical inference is made. This feature of characterization of probability distributions is peculiar to characterizing property and attracted attention of both theoretician and applied workers but there is no general theory of it.

Various approaches were used for characterization of negative exponential distribution. Among many other people, Fisz [1], Tanis [2], Rogers [3] and Fergusion [4] used properties of identical distributions, absolute continuity, constant regression of adjacent order statistics, linear regression of adjacent order statistics of random variables and characterized negative exponential distribution. Using independent and non-degenerate random variables Fergusion ([5,6]) and Crawford [7] characterized negative exponential distribution. Linear regression of two adjacent record values used by Nagaraja ([8,9],) were different from two conditional expectations, conditioned on a non-adjacent order statistics used by Khan [10] to characterize negative exponential distribution.

In this research note Section 2 is devoted for characterization based on identity of distribution and equality of expectation function randomly variable for a negative exponential distribution with probability density function (pdf).

*This work is supported by UGC Major Research Project No: F.No.42-39/2013(SR), dated 12-3-2013.

$$
f(x ; \theta)= \begin{cases}\mathrm{e}^{-(x-\theta)} ; & a<\theta<x<b \\ 0 ; & \text { otherwise }\end{cases}
$$

where $-\infty \leq a<b \leq \infty$ are known as constants, $\mathrm{e}^{-x}$ is positive absolute continuous function and $\mathrm{e}^{\theta}$ is everywhere differentiable function. Since derivative of $e^{\theta}$ is positive, the range is truncated by $\theta$ from left $\mathrm{e}^{-b}=0$.

\section{Characterization}

Theorem 2.1 Let $X$ be a random variable with distribution function $F$. Assume that $F$ is continuous on the interval $(a, b)$, where $-\infty \leq a<b \leq \infty$. Let $\phi(X)$ and $g(X)$ be two distinct differentiable and intregrable functions of $X$ on the interval $(a, b)$ where $-\infty \leq a<b \leq \infty$ and moreover $g(X)$ be non constant. Then

$$
E[g(X)-(\mathrm{d} / \mathrm{d} X) g(X)]=g(\theta)
$$

is the necessary and sufficient condition for pdf $f(x ; \theta)$ of $F$ to be $f(x ; \theta)$ defined in (1.1).

Proof Given $f(x ; \theta)$ defined in (1.1), for necessity of (2.1) if $\phi(X)$ is such that $g(\theta)=E[\phi(X)]$ where $g(\theta)$ is differentiable function then

$$
g(\theta)=\int_{\theta}^{b} \phi(X) f(x ; \theta) \mathrm{d} x
$$

Differentiating with respect to $\theta$ on both sides of (2.2), replacing $X$ for $\theta$ and simplifying one gets

$$
\phi(X)=[g(X)-(\mathrm{d} g(X) / \mathrm{d} x)]
$$


which establishes necessity of (2.1). Conversely given (2.1), let $k(x ; \theta)$ be such that

$$
g(\theta)=\int_{\theta}^{b}[g(x)-\mathrm{d} g(x) / \mathrm{d} x] k(x ; \theta) \mathrm{d} x
$$

which can be rewritten as

$$
\begin{aligned}
g(\theta)=-\mathrm{e}^{\theta} \int_{\theta}^{b}\left[\left(g(x)+\left\{\mathrm{e}^{-x} /\left(\mathrm{de}^{-x} / \mathrm{d} x\right)\right\}(\mathrm{d} g(x) / \mathrm{d} x)\right)\right. \\
\left.k(x ; \theta) /\left(-\mathrm{e}^{\theta}\right)\right] \mathrm{d} x
\end{aligned}
$$

which reduces to

$$
\begin{aligned}
g(\theta)= & -\mathrm{e}^{\theta} \int_{\theta}^{b}\left[\mathrm{~d}\left(\mathrm{e}^{-x} g(x)\right) / \mathrm{d} x\right] \\
& {\left[k(x ; \theta) /\left(-\mathrm{e}^{\theta}\right)\left\{\mathrm{d}\left(\mathrm{e}^{-x}\right) / \mathrm{d} x\right\}\right] \mathrm{d} x }
\end{aligned}
$$

Hence

$$
k(x ; \theta)=\mathrm{e}^{\theta}\left(-\mathrm{de}^{-x} / \mathrm{d} x\right) .
$$

Since $\mathrm{e}^{x}$ is increasing integrable and differentiable function on the interval $(a, b)$ with $\mathrm{e}^{-b}=0$ the following identity holds

$$
g(\theta) \equiv-\mathrm{e}^{\theta} \int_{\theta}^{b}\left[\mathrm{~d}\left\{\mathrm{e}^{-x} g(x)\right\} / \mathrm{d} x\right] \mathrm{d} x .
$$

Differentiating $\left\{\mathrm{e}^{-x} g(x)\right\}$ with respect to $x$ and simplifying (2.8) after taking $-\mathrm{de}^{-x} / \mathrm{d} x$ as one factor, (2.8) reduces to

$$
g(\theta)=\int_{\theta}^{b} \phi(x) k(x ; \theta) \mathrm{d} x,
$$

where $\phi(X)$ is a function of $X$ only derived in (2.3) and $k(x ; \theta)$ is a function of $x$ and $\theta$ only derived in (2.7).

Since $\mathrm{e}^{x}$ be increasing integrable and differentiable function on the interval $(a, b)$ where $-\infty \leq a<b \leq \infty$ and since $-\mathrm{de}^{-x} / \mathrm{d} x$ is positive intregrable function on the interval $(a, b)$ where $-\infty \leq a<b \leq \infty$ with $\mathrm{e}^{-b}=0$ and integrating (2.7) over the interval $(\theta, b)$ on both sides, one gets (2.7) as

$$
k(x ; \theta)=\mathrm{e}^{-\theta}\left(-\mathrm{de}^{-x} / \mathrm{d} x\right) ; a<\theta<x<b
$$

and

$$
1=\int_{\theta}^{b} k(x ; \theta) \mathrm{d} x .
$$

Substituting $\mathrm{de}^{-x} / \mathrm{d} x$ in $k(x ; \theta)$ derived in (2.10), $k(x ; \theta)$ reduces to $f(x ; \theta)$ defined in (1.1) which establishes sufficiency of (2.1).

Remark 2.1 Using $\phi(X)$ derived in (2.3), the $f(x ; \theta)$ given in (1.1) can be determined by

$$
M(X)=(\mathrm{d} g(x) / \mathrm{d} x) /(\phi(x)-g(X))
$$

and pdf is given by

$$
f(x ; \theta)=-(\mathrm{d} T(x) / \mathrm{d} x) / T(\theta)
$$

where $T(x)$ is decreasing function for $-\infty \leq a<b \leq \infty$ with $T(b)=0$ such that it satisfies

$$
M(X)=\mathrm{d}[\log \{T(X)\}] / \mathrm{d} X .
$$

Illustrative Example: Using method described in the remark characterization of negative exponential distribution through survival function $g(\theta)=\bar{F}(\theta)=\mathrm{e}^{-(t-\theta)}$ is illustrated.

$$
\begin{aligned}
& g(X)=\mathrm{e}^{-(t-X)} \\
& \phi(X)=g(X)-\mathrm{d} g(X) / \mathrm{d} X=\mathrm{e}^{-(t-X)}-\mathrm{e}^{-(t-X)}=0 \\
& M(X)=(\mathrm{d} g(X) / \mathrm{d} X) /(f(X)-g(X))=-1 \\
& \mathrm{~d}\left\{\log \left(\mathrm{e}^{-X}\right)\right\} / \mathrm{d} X=M(X)=-1 \\
& \therefore T(X)=\mathrm{e}^{-X} \\
& f(x ; \theta)=\mathrm{e}^{-(x-\theta)} ; a<\theta<x<b
\end{aligned}
$$

\section{Conclusion}

To characterize pdf defined in (1.1) one needs any arbitrary non-constant function of $X$ which should only be differentiable and integrable.

\section{REFERENCES}

[1] M. Fisz, "Characterization of Some Probability Distributions,” Skandinavisk Aktuarietidskrift, Vol. 41, No. 1-2, 1958, pp. 65-70.

[2] E. Tanis, "Linear Forms in the Order Statistics from an Exponential Distribution," The Annals of Mathematical Statistics, Vol. 35, No. 1, 1964, pp. 270-276.

[3] G. S. Rogers, "An Alternative Proof of the Characterization of the Density AxB," The American Mathematical Monthly, Vol. 70, No. 8, 1963, pp. 857-858.

[4] T. S. Ferguson, "A Characterization of the Negative Exponential Distribution,” The Annals of Mathematical Statistics, Vol. 35, 1964, pp. 1199-1207.

[5] T. S. Ferguson, "A Characterization of the Geometric Distribution,” The American Mathematical Monthly, Vol. 72, No. 3, 1965, pp. 256-260.

[6] T. S. Ferguson, "On Characterizing Distributions by Properties of Order Statistics,” Sankhyā: The Indian Journal of Statistics, Series A (1961-2002), Vol. 29, No. 3, 1967, pp. 265-278.

[7] G. B. Crawford, "Characterizations of Geometric and Exponential Distributions,” The Annals of Mathematical Statistics, Vol. 37, No. 6, 1966, pp. 1790-1795.

[8] H. N. Nagaraja, "On a Characterization Based on Record Values,” Australian Journal of Statistics, Vol. 19, 1977, pp. 70-73.

[9] H. N. Nagaraja, "Some Characterization of Continuous Distributions Based on Adjacent Order Statistics and Re- 
cord Values,” Sankhy, Series A, Vol. 50, No. 1, 1988, pp. 70-73.

[10] A. H. Khan, M. Faizan and Z. Haque, "Characterization of Probability Distributions through Order Statistics,” Prob Stat Forum, Vol. 2, 2009, pp. 132-136. 